\title{
The contributions of occupational science to the readiness of long duration deep space exploration
}

\author{
Janis Davis*, Macy Burr, Maria Absi, Rochelle Telles and Howard Koh \\ Occupational Therapy Program, Stanbridge College, Irvine, CA, USA
}

Received 22 November 2015

Accepted 3 May 2016

\begin{abstract}
.
BACKGROUND: This study introduces the contributions of occupational science (OS) to the preparation and support of astronauts during long duration space exploration. Given the hostile environment of space, it is not surprising that there is grave deterioration of both physical and mental health when off Earth. However, OS, through occupational therapy (OT), can identify strategies that maintain health and minimize disruptions in task performance for mission success.

OBJECTIVE: To determine the gaps in NASA's preparation of astronauts for long duration space exploration and the viable contributions of OT. Because occupational therapists are trained to address deficits and modify environments to support meaningful engagement in occupations, the OT practitioner is well suited to address the disabling conditions astronauts experience in space.

METHODS: A literature review revealing the challenges of deep space travel on humans was completed. A survey was also sent to $(\mathrm{N}=170)$ occupational therapists worldwide to identify opinions about the profession's involvement in deep space exploration.

RESULTS: Ninety-seven percent $(\mathrm{N}=163)$ of the participants believed that OS can inform long duration space travel. Approximately ninety-eight percent $(\mathrm{N}=166)$ of respondents believed that OT interventions can be used on space travelers during long duration space flights.

CONCLUSION: OT interventions can be implemented in any phase of space flight to increase the likelihood of mission success and astronaut safety and well-being.

Keywords: Occupational therapy, meaning, astronaut, health effects, Mars Mission, gravity

\section{Introduction}

Long duration space exploration places humans in a unique environment never before experienced by earthbound souls. A significant body of research currently exists describing the various health effects of space travel on astronauts. Scientific knowledge and technology such as magnetic resonance

\footnotetext{
*Address for correspondence: Janis Davis, PhD, OTR/L, Stanbridge College, 2041 Business Center Drive, Irvine, CA 92612, USA. Tel.: +1 949794 9090/Ext. 5282; Fax: +1 949794 9094; E-mail: Janis.davis@stanbridge.edu.
}

imaging (MRI), computed tomography (CT) scans and positron emission tomography (PET) scans of focal areas in the body have allowed researchers to distribute the body into subcomponents to study the effects of space exploration on human health [1-8]. Unfortunately, space travel is not commonly viewed from a holistic and occupation-based perspective. This study is unique in that it sought to identify how Occupational Therapy (OT), with its foundation in Occupational Science (OS), could contribute to the well being and optimized task performance of astronauts.
\end{abstract}


Occupational science is multidimensional with its philosophy grounded in a holistic view of the individual. The science studies the components of function as well as occupations, encompassing the elements of cognition, psychomotor skills, emotions, social awareness, self-knowledge, and many other concepts associated with a variety of social science and health disciplines. Occupational science is primarily concerned with promoting health, well-being, and a higher quality of life through a balance of occupations. It is also used to inform all areas of OT practice [9]. By examining the transactional relationship between the individual (client factors) and skills needed for optimal task performance (performance skills), occupational therapy practitioners facilitate health, well-being, and enhanced participation in life's roles [10].

This transactional relationship between occupation and overall health have been investigated by numerous researchers [11-14]. For example, Shaw and Rudman [11] have explored occupational science concepts and their impact on health, well-being and productivity. Trudela et al. [14] discussed the link between a stressful work environment and psychological stress. Furthermore, Schreuer, Myhilla, Aratan-Bergman, Samant, \& Blanck [13] claim that occupational therapists play a unique role in the workplace environment to optimize productivity and task performance of workers who may benefit from ergonomic accommodations.

Given the extreme conditions in space (e.g. microgravity, radiation, isolation), there is degradation in many health domains that will influence well-being and occupational performance [1-8]. Occupational therapy practitioners are uniquely qualified to examine the intersection of person, environment, occupation, and performance during long duration space exploration through current practice models and frames of reference such as psychodynamic, cognitive behavioral, neurodevelopmental, biomechanical and sensory integration. A thorough review of the literature was undertaken to reveal the risk factors associated with space travel related to human health and functioning. In addition, ideas regarding interventions to promote wellness for life in space to ameliorate these risks were explored.

Another purpose of this study was to determine if occupational therapy clinicians and educators would agree that OT has a place in long duration space exploration. The researchers were also interested in what types of models, frames of reference, and interventions OT clinicians and educators would use to contribute to the health and well being of astronauts.

\section{Method}

\subsection{Participants and procedures}

Given the exploratory nature of this study, a mixed method approach was used. A literature review was conducted to identify domains of human health and performance compromised during space travel. In addition, a survey was sent to OT practitioners worldwide to further investigate occupational therapists' opinions regarding the subject and to suggest potential interventions.

The literature review was limited to Englishlanguage literature published primarily between 1990 and 2015. Databases were searched including: Google Scholar and EBSCO host including: Medline Complete, ERIC, Ebook collection and CINAHL complete. Search terms used included: "National Aeronautics and Space Administration (NASA)", "space exploration", "neuroscience", "psychological effects", "cardiovascular effects", "astronaut", "cognition", "physiological effects", "confinement", "isolated", "cognitive neuroscience", "context", "environmental conditions", "occupational deprivation", "sleep deprivation”, "space, "sensory deprivation", "neurocognitive performance", "incapacitating symptoms", "cardiac", "human", "sympathetic nervous system", "life science", "space flight", "space travel", "health effects", "immune response", "psychological stress", "health consequences", "stress" "workers" Basic inclusion criteria were publications dating primarily between 1990 to 2015, and articles pertaining to the domains of health OT practitioners might be concerned with that are affected by space travel (See Fig. 1). A drop-box with news articles, fact sheets, and various resources provided by the Long Duration Space Exploration Behavioral Readiness Consortium, 2030 (DSR30) and advisement from American astronaut, Dr. Yvonne Cagle, was used to guide research and draw conclusions.

After approval from the Institutional Review Board for Protection of Human Subjects, researchers developed an eighteen-item questionnaire to send to occupational therapy practitioners and educators. Demographic questions such as area of practice, education level, age, and area of residence were included. Questions regarding what types of OT frameworks, 


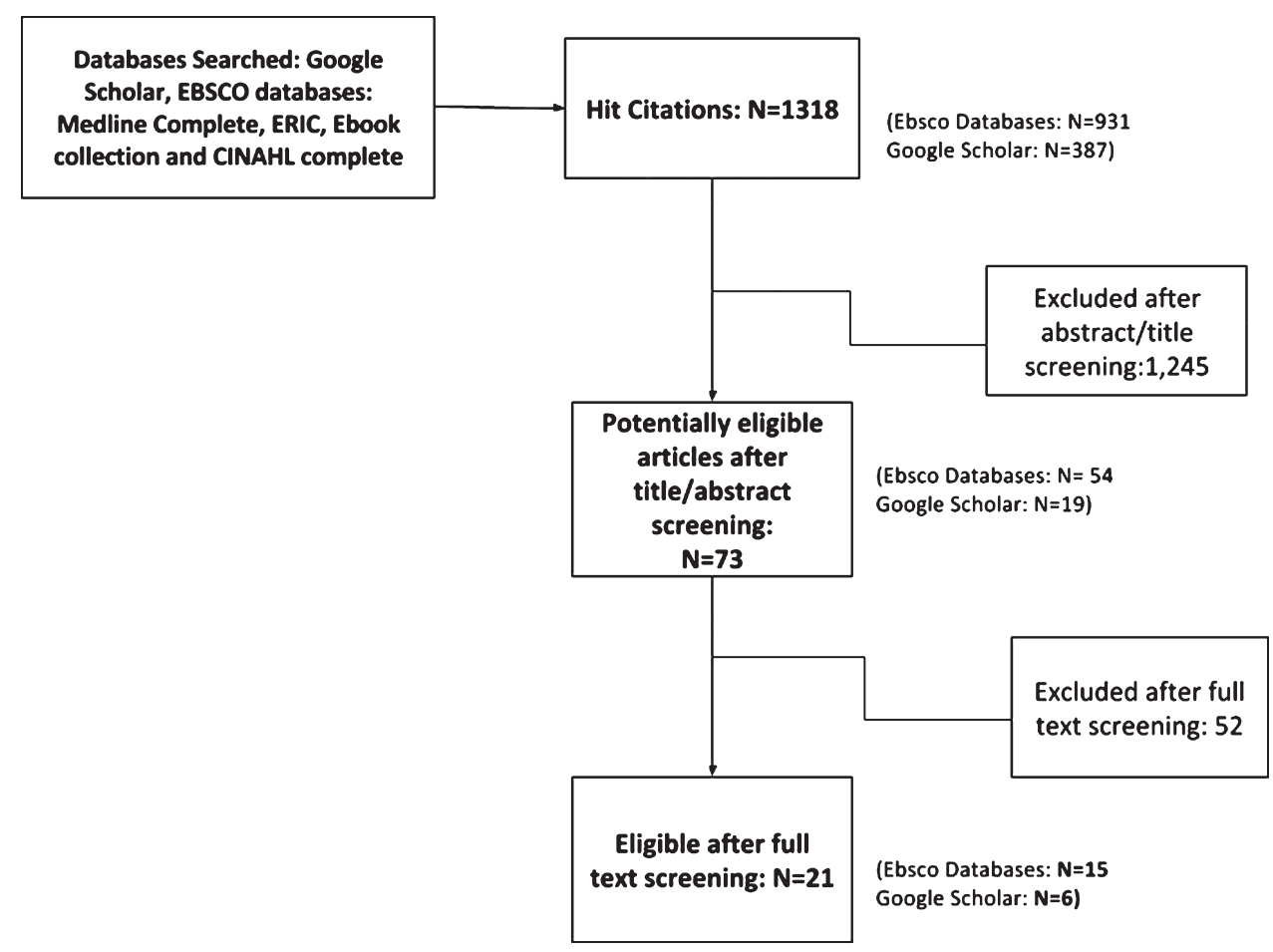

Fig. 1. The Literature Review Process for the Investigation of Domains of Human Health Affected by Space Travel.

models and interventions were included. Open-ended questions regarding thoughts about how OS and OT could contribute to long duration space travel were included. Random and snowball sampling was used to gather email addresses from OT practitioners and educators. The survey was also circulated to OT practitioners in California through the Occupational Therapy Association of California (OTAC) email list. Participants were encouraged to send the survey to people within their professional networks. Google Docs was used to send the anonymous survey and completion of the survey indicated consent. If the participant wished to learn of the survey results the option was given to include their contact information upon completion of the survey. The analysis tool in Google Docs provided the analytics for the quantitative information.

\subsection{Analysis}

Results of the qualitative information were coded to identify common themes and sub-themes. Each researcher read participant's responses thoroughly. Categories were identified and headings selected. Under each heading, phrases or words were listed. Further examination of the data yielded themes and subthemes by searching for patterns and connections among responses. To establish interrater reliability, each researcher coded the information until common themes and subthemes were identified [11-13]. The findings revealed there is a high level, excellent, agreement among raters, $k=0.90$.

\section{Literature review results}

Occupational therapists are trained in assessing the interrelated components of client factors such as body functions and body structures when considering a client's ability to engage in meaningful occupations. Body structures include anatomical parts of the body which support body functions [10]. Body functions which are dependent upon body structures include sensory, affective, cognitive, perceptual, cardiovascular, respiratory and endocrine functions [10]. Because astronauts engaged in long duration space exploration need to adapt to a hostile anti-gravity environment, are exposed to radiation, perform under extreme stress, and are essentially isolated from the rest of humanity, there are many detrimental effects imposed on them. Table 1 displays the 
Table 1

Client factors affected by space travel

\begin{tabular}{|c|c|}
\hline Client Factor & Disruptions \\
\hline Body Structures & $\begin{array}{l}\text { Bone mineral loss, skeletal muscle atrophy, } \\
\text { cardiovascular deconditioning, radiation } \\
\text { effects on neuroplasticity, cerebral-spinal } \\
\text { fluid compressing on the optic nerve }\end{array}$ \\
\hline Body Functions & $\begin{array}{l}\text { Alterations in muscle activation variability, } \\
\text { orthostatic reflexes, decreased strength, } \\
\text { balance problems, vision degeneration, } \\
\text { sensory deprivation, the lack of sensory } \\
\text { integration needed for optimal functioning } \\
\text { including proprioception, sight, auditory } \\
\text { and tactile restrictions, impaired manual } \\
\text { control, impaired executive functioning, } \\
\text { concentration problems, anxiety, } \\
\text { depression }\end{array}$ \\
\hline
\end{tabular}

client factors affected by space travel. Furthermore, Table 2 shows the performance skills that are impaired by the extreme environment experienced in space.

\subsection{Cardiovascular effects}

Space travel has extreme adverse effects on the cardiovascular system [3]. In the beginning of space flight the central blood volume increases while perfusion and hydrostatic pressure in the lower half of the body decreases. The slightly higher preload and stroke volume can lead to bradycardia, increased renal blood flow, and polyuria. Over time the plasma volume and the efficacy of orthostatic reflexes regulating blood pressure decrease. These effects can lower blood pressure, cause fainting, and make it difficult to stand upright. Other cardiovascular changes such as heartbeat and heart rhythm irregularities and reduced aerobic capacity may occur [3].

\subsection{Musculoskeletal effects}

Researchers have revealed that the anti-gravity environment also has negative effects on the musculoskeletal system [3]. Specifically, skeletal muscle atrophy is a major concern [4]. Research indicates that long-term missions could reduce overall muscle function by nearly half [2]. Study subjects who completed missions with duration of up to two weeks showed alterations in muscle activation variability. Specifically, increased variability in ankle and knee joint motion, alterations in head-trunk coordination and impaired balance were noted. Osteoporosis is also a serious health consequence caused by the loss of mechanical stress experienced in space $[2,5]$. Canan [2] indicated there to be a continuous and progressive bone loss during microgravity exposure that can be identified as early as after one week in space. Furthermore, Fulford [5] found that the leg and hip bones lose their density most quickly in microgravity increasing the risk of: kidney stones, fractures, hip and spine issues and impaired healing ability.

\subsection{Sensorimotor impairments}

In addition to the debilitating effects of low gravity on the cardiovascular and musculoskeletal system, radiation exposure may cause central nervous system damage. Researchers have found that changes in the sensorimotor system occur during space travel [8]. These impairments include: diminished control of movement, reduced ability to see and interpret information from the eyes, impaired spatial orientation, and difficulty walking [4]. Alterations in the sensorimotor system can contribute to impaired postural control, locomotion, gait and manual control [4, 8].

Table 2

Performance skills affected by space travel

\begin{tabular}{lc}
\hline Performance Skills & Disruptions \\
\hline Motor and Praxis Skills & $\begin{array}{c}\text { Delayed motor controls and spatial orientation leading to significant challenges completing tasks and } \\
\text { meaningful occupations, motor coordination and movement timing problems, impaired postural }\end{array}$ \\
& control and self position accuracy, difficulty walking \\
Vision degeneration, sensory deprivation, the lack of sensory integration needed for optimal \\
functioning including proprioception, sight, auditory and tactile restrictions, visual attention \\
problems, somatosensory problems, sound localization difficulties, vestibular dysfunction and \\
binaural hearing \\
Color perception, memory loss, impaired executive functioning, diminished attention, perceptual \\
disorientation \\
Emognitive Skills & Psychological effects of extreme working conditions, excessive activation of the stress response, \\
depression, irritability, and lack of motivation. & Ineffective communication, lack of team cohesion, interpersonal conflict, diminished social \\
Communication Skills & interaction
\end{tabular}


Astronauts can also suffer from an overall perceptual distortion, disorientation and reduced performance in tasks relying on a high level of sensory-motor and sensory-cognitive skills such as piloting a spacecraft.

\subsection{Cognitive deficits}

Research supports the relationship between microgravity exposure and reorganization in the somatosensory cortex and the cerebellum. For example, a decreased number of synapses and degeneration of axon terminals have also been identified [4]. De La Torre [3] suggest that the following neurocognitive deficits may occur: somatosensory problems, selfposition accuracy problems, color perception and loss of acuity, sound localization and binaural hearing, impaired executive functioning, concentration problems, difficulty acquiring targets in voluntary movements, visual attention problems, irritability, and lack of motivation.

\subsection{Vision degeneration}

Visual deficits have been identified as a major concern for astronauts undergoing space travel $[3,18]$. This can be attributed to structural changes in the retina and optical abnormalities caused by cerebral-spinal fluid compressing on the optic nerve [18]. Cowing [18] reported that about $29 \%$ of astronauts who returned from two-week space shuttle missions and $60 \%$ who spent six months aboard the International Space Station (ISS) reported visual deficits.

\subsection{Vestibular dysfunction}

The microgravity environment also disrupts the vestibular system. Motion sickness occurs because sensory input identified from the eyes, muscles, and joints or vestibular systems conflict with each other to cause these phenomena [3]. De La Torre [3] indicated that approximately $70 \%$ of astronauts experience space motion sickness during the first week of the mission. Astronauts also can suffer from a loss of balance and spatial orientation due to the vestibular system degrading [2].

\subsection{Sensory deprivation}

Because astronauts have restrictions of the visual, auditory, olfactory, kinesthetic, tactile and gustatory systems, they might be at risk for experiencing sensory deprivation [1]. Additionally, many aspects of space travel such as eating the same foods, hearing the same sounds, continuously smelling the same smells and being around the same people for such long periods of time may become monotonous. Researchers have suggested that a lack of sensory input can impair how information is processed in the brain [1]. Furthermore, Rasmussen [19] linked sensory deprivation to neurological changes in the brain that can lead to psychological effects such as hallucinations and anxiety.

\subsection{Sleep deprivation}

Circadian rhythms help maintain sufficient periods of sleep and wakefulness enabling people to perform occupations efficiently [3, 20, 21]. However, this system is driven by variable amounts of daylight and darkness in different seasons and places [3, 20, 21]. In space, the lack of environmental cues such as natural light and other factors such as anxiety, workload, stress and isolation result in sleep loss, poor quality of sleep, and fatigue in astronauts. Astronauts usually have between 5 to 6 hours of sleep per night, and even less in emergency situations [3]. Sleep deprivation is a major risk factor for astronauts because fatigue in the workplace has been associated with increased risk of accident and injury in several studies $[20,21]$.

\subsection{Physical and psychological effects of stress}

Stress is also a risk factor for astronauts undergoing long duration space travel that has physiological and psychological implications [22]. Not only do daily stressors lead to irritability, short temperedness, and a lack of concentration, but psychological stress can have long-term effects on an individual's sympathetic nervous system [7, 9]. Psychological stress has been described as an adaptation to the fight or flight response [23]. With excessive activation of this response, elements such as cortisol, catecholamines and neuropeptides can produce negative effects on the immune system and can possibly contribute to disease onset and progression [23]. Extreme stress can even induce cardiac arrhythmias [24]. Stressors in space that might trigger this response include: constant acceleration, heat, noise, vibration, problems with food and oxygen supply or waste disposal, radiation, and toxic fuels [7]. NASA Extreme Environment Mission Operations (NEEMO) has indicated 
the psychological effects include depressed mood, anger, irritability, anxiety, interpersonal tension and conflict with group members [6].

\subsection{Interpersonal conflict and ineffective communication}

Currently only analogue missions such as NEEMO, the inflatable lunar habitat in Antarctica, and other research programs have been set in place to explore how humans tolerate and interact in deprived environments. In the polar analogue mission, group interaction, outside communication, workload, and recreation/leisure were found to be the most salient behavioral issues [25]. Interpersonal conflict and ineffective communication is a major risk factor during space exploration. A crew that cannot work together in a cohesive manner will have negative effects on occupational performance and mission success.

\section{Occupational therapy's analogous contexts and interventions}

The Occupational Therapy Practice Framework (OTPF) which outlines the domain and process of the profession of occupational therapy lists the client factors and performance skills needed to perform most occupations [10]. It also outlines the types of interventions used in therapy to remediate or sustain optimal health and wellness. Occupational therapy practitioners routinely work with persons with neurological, central nervous system, visual, musculoskeletal, cardiac, vestibular, sensory and mental health disorders. Therefore, occupational therapy practitioners may have a role in addressing these deficits, and enabling astronauts to work effectively and efficiently on long duration space exploration missions.

Occupational therapy practitioners have been identified as important members of the cardiac rehabilitation team [26]. Not only do they assist their clients in returning to meaningful activity and participation in life roles, but they also have the knowledge and background to address psychosocial adjustment issues. For example, OT practitioners teach patients with cardiovascular disorders relaxation and control of breathing techniques that ultimately help improve their symptoms [27]. They also play a key role in noticing defense mechanism like denial, and educating the client and family on coping strategies to mitigate the fear and anxiety that often accompanies a myocardial infarction.

Occupational therapy practitioners also have extensive knowledge regarding the musculoskeletal system, and how the body produces movement to achieve wanted motor tasks. This suggests that therapists who specialize in working with clients with musculoskeletal disorders might offer insight into how astronauts can best perform required duties while dealing with the consequences of low gravity. By helping astronauts increase range of motion, strength and endurance, OT practitioners can facilitate optimal performance.

Additionally, neurodevelopmental techniques may be beneficial in helping astronauts retain normal movement. Occupational therapy practitioners utilizing these techniques help their clients to restore normal movement, and functioning, by providing sensory information regarding natural muscle activation patterns. Researchers have even found that pressure on the soles of an astronaut's foot can promote a vertical sense and increase neuromuscular activation, suggesting the effectiveness of interventions based on neurodevelopmental theory [8].

When planning for long duration space exploration, occupational therapists would suggest sensorimotor interventions. Helen Cohen, an occupational therapist, was part of a team that developed a training program aimed to improve the ability of astronauts to adapt to new sensory environments called the Sensorimotor Adaptability (SA) Training [28]. By assisting crew members in learning how to solve sensorimotor, balance, and/or locomotor challenges, adaptation to this extreme environment can be increased. Sensorimotor Ability Training involves walking on a treadmill that is mounted on a six degree of freedom motion base in front of a screen that provides visual stimuli. It has been proven to improve locomotor adaptability, and stability, which reduces energy expenditure [28]. Ironically, one of the very few occupational therapists involved with NASA is involved in this training program.

Occupational therapy experts in vestibular rehabilitation offer interventions to reduce the negative effects that anti-gravity has on the vestibular system. Motion sickness has been controlled by a user-worn see-through display, utilizing a visual fixation target coupled with a stable artificial horizon, and aligned with user movement for people experiencing motion intolerance and spatial disorientation [29]. Vestibular rehabilitation training has also been shown to improve other symptoms that are associated with 
space travel [30]. For example, Porciuncula, Johnson, \& Glickman [30] identified vestibular rehabilitation training as an effective means of improving postural stability and gaze. Additionally, Cohen and Kimball [32] found that a vestibular rehabilitation program was effective in decreasing vertigo and improving independence in daily living. Cohen [34] asserts that occupational therapists play a vital role in vestibular rehabilitation because many patients don't follow their exercise regimens, and it might be more effective to incorporate vestibular rehabilitation exercises into functional activities [33]. Interestingly, the Vestibular Disorders Activities of Daily Living Scale (VADL), was created by an occupational therapist, and may be a helpful instrument in determining how vestibular dysfunction is interfering with functional abilities of astronauts [34].

Vestibular functioning is only one of the few sensory impairments OT practitioners are skilled in addressing. Therapists practicing in vision rehabilitation help their clients remain as independent as possible, while dealing with the functional limitation that comes along with vision impairment. For example, they teach their clients how to compensate for visual acuity loss by using adaptive equipment or techniques to enhance functioning [35]. They determine how vision impairment has limited the person's ability to complete tasks, and then modify the task and/or the environment accordingly. Furthermore, OT practitioners also apply their expertise with adaptive devices and assistive technology to enable people to use optical and non-optical devices to complete activities [35]. As experts in activity analysis and environmental modification, occupational therapists are in a unique position to address vision problems experienced in space.

Because occupational therapists are trained to address deficits and modify environments to support meaningful engagement in occupational endeavors, they have insight into ways the spacecraft can be modified to reduce sensory deprivation. Bachmand et al. [1] have revealed the influence of sensory input on cognition and the way in which information taken in from the environment influences information processed in the brain. The Biophilia hypothesis proposes that individuals have evolutionarily adapted to gather information through exploring their environment using the senses, and this curiosity has been beneficial to the development of cognitive processes [1]. This implies that a lack of sensory input experienced on a space mission can have a negative impact on cognition. Mansfield-Cohen et al. [36] identified the positive effects that multisensory environments have on mood levels and cognition suggesting the importance of enhancing the environment of the spacecraft. Implementing a variety of stimuli can be beneficial to an astronaut's cognition. For example, video screens and virtual reality headsets may be useful modalities. Specifically, videos of hikes, trails or landscapes could make exercising on the treadmill more enjoyable [1]. Three-dimensional video games or videos could also offer crew members ways to explore the environment and increase sensory input [1].

Cognitive rehabilitation may be necessary for astronauts experiencing the cognitive deficits induced by space travel. Occupational therapy practitioners who specialize in working with neurological conditions such as dementia, cerebrovascular disease, or traumatic brain injuries could offer cognitive training regimens to maintain or improve the problem-solving skills required of astronauts to complete mission tasks. Researchers have found that repetitive cognitive training can result in macro and micro structural activity changes in the brain as identified by an MRI in people with brain injuries [37]. Additionally, Cho et al. [38] indicated that cognitive enhancement training improves the performance of daily living activities and cognition and decreases depression levels in older adults with dementia [38]. Similarly, Cochet et al. [39] identified cognitive remediation to be effective in improving problem solving, memory and attention in individuals with schizophrenia. Because cognitive training has been identified as an appropriate countermeasure in addressing cognitive deficits in a wide range of populations, there are many implications for the role that OT practitioners who work in cognitive rehabilitation might play in addressing the cognitive deficits that astronauts experience in space.

OT interventions focused on stress management may provide opportunities for astronauts to deal with the dynamic interactions of mental, physical and psychological stressors associated with the astronaut experience. Occupational therapists practicing in a psychosocial setting are highly skilled in helping clients overcome anxiety by teaching relaxation techniques and self regulation skills to recognize and manage their emotions, thoughts, and behaviors. Smith-Forbes et al. [40] describes occupational therapy's role in addressing intellectual, physical, emotional and or behavioral reactions of service members in combat or other military operations. Stressors during combat may mirror 
stressors experienced in space, suggesting the need for OT interventions in optimizing occupational performance of astronauts. Training astronauts on the use of cognitive behavioral techniques, such as cognitive restructuring, may assist in minimizing the irrational thinking that could occur during long duration space exploration.

Sleep deprivation may be even more debilitating than operating under extreme stress. Fatigue in the workplace has been associated with increased risk of accident and injury in numerous studies [20, 21, 41]. However, by modifying performance patterns such as habits, roles and routines, OT interventions can address the sleep problems experienced by astronauts. Sleep and rest are now its own area of occupation in the American Occupational Therapy Association's practice framework [10]. Occupational therapists are trained to identify contextual, performance pattern activity demands and client factors that may interfere with restful sleep. Occupational therapy practitioners use stimulus control to address sleep problems such as adapting nightly routines to decrease stimuli [42]. They also incorporate sleep restriction/compression (e.g., setting a predetermined sleep schedule) techniques such as sleep education, light therapy and relaxation techniques. [42] Additionally, OT interventions to promote better sleep can include physical activity, earplugs, eye-masks, and sleep inducing music [42].

Ball [25] states that the highest priority in optimizing performance and general living conditions will be the development of an effective systematic approach to the management of a compatible productive team, who must live together in an isolated dangerous environment. Selection of personnel will be strenuous, requiring those intended to travel into deep space for longer periodic missions, in order to go through a rigorous screening process. Not only are OT practitioners trained to identify barriers to optimal occupational performance at the personal level, but also on a social level. For example, group work is a common treatment modality used by occupational therapists practicing in psychiatric settings [43]. In utilizing this treatment approach, occupational therapists must assess culture and societal factors that influence roles and group behavior. Because most OT practitioners are mandated to be culturally competent, they are knowledgeable about group leadership and dynamics. This implies that they may have insight into developing crews that can work together efficiently.

\section{Results of the survey}

Occupational therapists work in a variety of setting, some traditional, like hospitals and schools, and some emerging practice areas like oncology and homelessness. This research team was interested in finding out what OT practitioners would think about the OT role in long duration space exploration. From the results of the online based survey, $96 \%$ of the participants believed that OS can inform long duration space travel. $97.6 \%$ of respondents believed that OT interventions can be used on space travelers during long duration space flights. When given a list of OT frames of reference, and asked which ones would specifically contribute to a long duration space mission, $69 \%$ responded with biomechanical, $67 \%$ responded with sensory integration, $64 \%$ responded with cognitive behavioral, 58\% chose psychodynamic, $32 \%$ selected rehabilitation, $20 \%$ chose neurodevelopmental, $21 \%$ selected that another frame of reference could be used that was not mentioned, and $3 \%$ were unsure of a theoretical frame of reference that could be used (See Fig. 2). The following question on the survey stated, "What performance skills are most important to focus on while providing an OT intervention to astronauts?" The responses included: $80 \%$ emotional regulation Skills, 64\% motor and praxis skills, 64\% sensory perceptual Skills, $46 \%$ cognitive skills, $35 \%$ communication, and $10 \%$ said other. The last question with detailed data asked participants to select an OT model that they thought would contribute to long duration space travel. The answers were as followed: $66 \%$ person-environment-occupational-performance model (PEOP), 57\% model of human occupations (MOHO), $41 \%$ occupational adaptation, 33\% ecology of human performance model, $24 \%$ occupational behavior model, $13 \%$ unsure, and $7 \%$ said other (See Fig. 3).

\section{Discussion}

Inherent in the OS discipline is the link between engagement in meaningful activity and well-being. Therefore, it could have been expected that the one concept that came up most frequently in the participant's qualitative responses (62 out of 170 or approximately $37 \%$ of participants) was meaning (See Table 3). With the inability to participate in meaningful occupations, well-being is threatened. This implies that continued participation in 


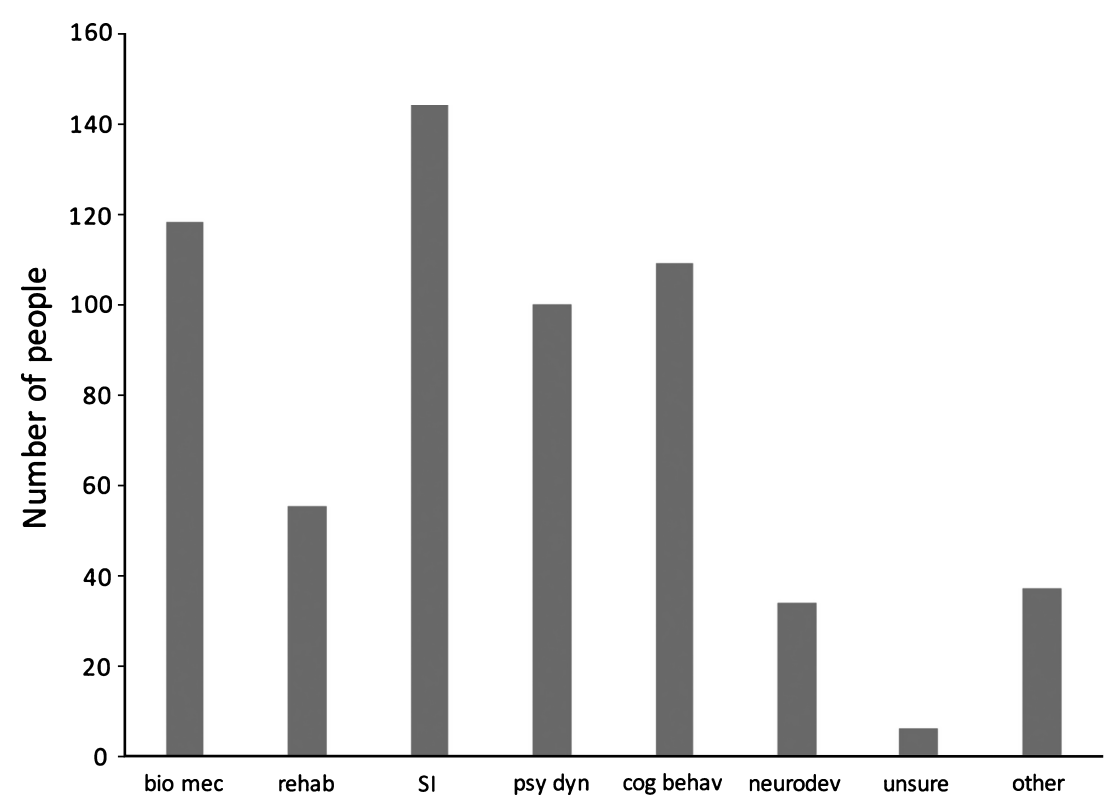

Fig. 2. Occupational therapy frames of reference with potential to contribute to a long duration space mission. Survey responses to "What specific occupational therapy frames of reference could contribute to a long duration space mission?" Bio $\mathrm{mec}=$ biomechanical, rehab = rehabilitation, $\mathrm{SI}=$ sensory integration, psy dyn = psychodynamic, $\operatorname{cog}$ behav $=$ cognitive behavioral and neurodev $=$ neurodevelopmental.

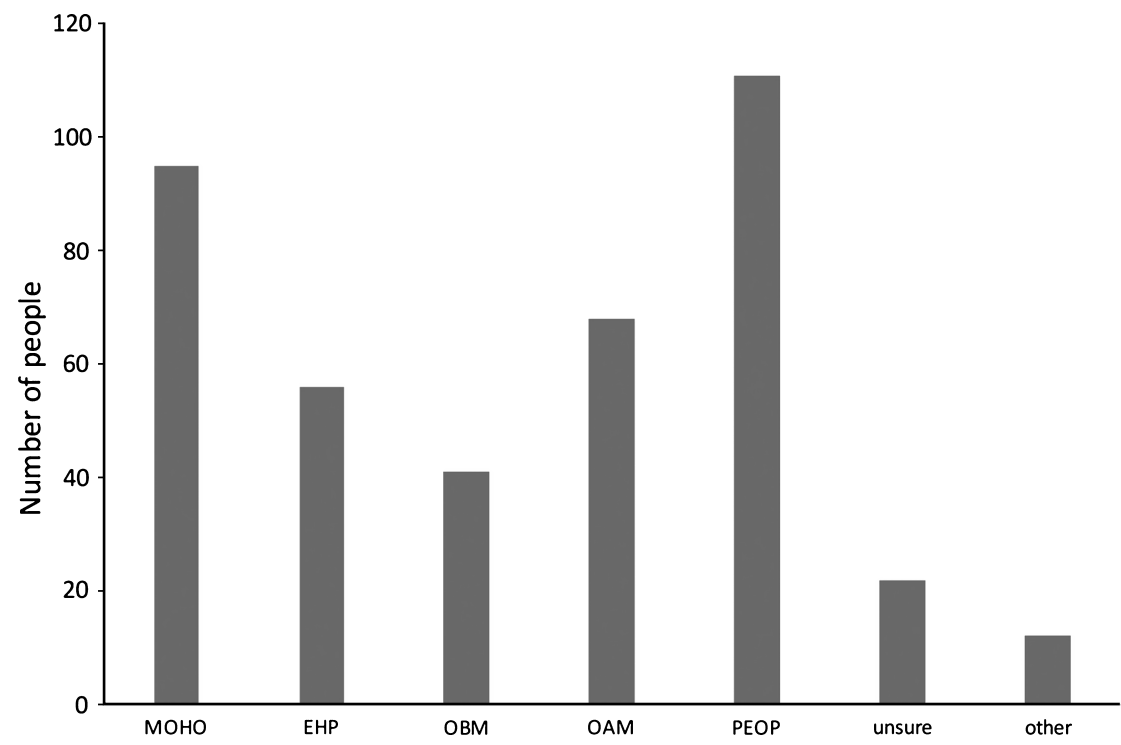

Fig. 3. Occupational therapy models for long duration space missions. Survey responses to "Which of the following OT models do you think can contribute to long duration space travel?" MOHO = Model of Human Occupation, EHP = Ecology of Human Performance, $\mathrm{OBM}=$ Occupational Behavior Model, OAM = Occupational Adaptation Model, PEOP = Person- Environment- Occupational- Performance Model.

meaningful occupations, in addition to work, is crucial to optimize the health of astronauts.

While considering that astronauts will be undergoing long duration space exploration, leisure may be subordinate to the completion of mission objectives.
However, leisure might be a necessary means of restoration. Leisure was mentioned 51 times by occupational therapists (see Table 3). From an OS perspective, leisure is crucial for optimal health and well-being. Numerous researchers have revealed the 
Table 3

Thematic coding of survey fill in responses

\begin{tabular}{lc}
\hline Themes with subthemes & Frequency $(f)$ \\
\hline Meaning & 62 \\
Psychosocial Interventions & 52 \\
Coping with Stress/Depression & 23 \\
Emotional Regulation & 8 \\
Cognitive Training/Retraining & 7 \\
Mindfulness/Awareness Training & 6 \\
Journaling/ Storytelling & 4 \\
Leisure & 51 \\
Sensory-Perceptual Interventions & 48 \\
Integration & 16 \\
Proprioception & 5 \\
Vestibular & 4 \\
Modulation & 2 \\
Biomechanical Interventions & 40 \\
Strengthening & 14 \\
Physical Activity & 12 \\
ROM/Stretching & 8 \\
Ergonomics & 8 \\
Endurance & 2 \\
Isometrics & 2 \\
Adaptation & 36 \\
Communication and Social Skills & 31 \\
Maintaining Social Connections & 12 \\
Group Interaction & 9 \\
Social Skills & 5 \\
Performance Patterns & 30 \\
Routines & 21 \\
Roles & 7 \\
Rituals & 3 \\
Habits & 3 \\
Occupational Balance & 10 \\
Occupational Science & 19 \\
\hline
\end{tabular}

The fill-in survey responses for "I believe the following OT interventions may be useful for space travelers who endure long periods off Earth," "what other thoughts do you have about the role of Occupational Science in deep space exploration?" and "what other thoughts do you have about occupational therapy's role in deep space exploration?" were combined and thematically coded and reflect high interrater reliability. Note: Frequency is the total number of responses but unique to only one respondent.

positive effects of leisure activity on health and wellbeing [44, 45]. Specifically, Kleinberg et al. [45] identified leisure as an important resource for dealing with stressful life events. Similarly, Garcia et al. [46] identified the link between leisure-time physical activity and psychological well-being, which was operationally defined as self-esteem and subjective vitality. Because humans have an intrinsic need for contact with nature, horticultural leisure activities might be especially beneficial and might even address sensory deprivation $[44,47]$. Some occupational therapists who responded to the survey recommended virtual reality games with nature-scenes and the utilization of plants on the spacecraft. Future research should focus on enabling astronauts to participate in an optimal amount of restorative leisure time. Additionally, ways to maintain occupational balance should be investigated.

Many OT practitioners recommended interventions that aligned with the gaps in knowledge identified by NASA on the Human Research Roadmap. For example, psychosocial interventions were mentioned 52 out of 170 times (See Table 3). Occupational therapy practitioners employing psychosocial techniques could make suggestions for closing this gap and identify and validate effective treatments for adverse social conditions and psychiatric disturbances that might occur during the mission [48]. Specifically, interventions focusing on coping with stress, depression and other behavioral consequences, emotional regulation, mindfulness and meditation were frequently mentioned (see Table 3).

The majority of therapists responding to the survey believed sensory perceptual skills and the sensory integration frame of reference to be of most importance while providing treatment to people traveling to space. They believed that sensory deprivation, sensory integration, proprioceptive and vestibular exercises would be important. Because sensory and sensorimotor deficits have been identified as gaps in knowledge to be addressed by NASA, OT assessments and interventions might provide an opportunity for them to be addressed.

Due to the extensive knowledge regarding motor and praxis skills in regards to human performance, OT practitioners likely have the background to assist NASA with creating interventions to treat musculoskeletal injuries that may occur during the mission [48]. They also might provide some insight into creating exercise regimens that assist in optimizing musculoskeletal health for maintenance of occupational performance. Approximately $64.1 \%$ of the participants felt that interventions focused on motor and praxis skills and the biomechanical frame of reference were significant while preparing for long duration space exploration. Specifically, physical activity, isometric exercises, range of motion (ROM) endurance and ergonomics appeared as major subthemes.

Team function has been identified as an important area to research by NASA. Additionally, understanding how personal relations/interactions affect behavioral health and performance is an area of concern [48]. Because occupational therapists have knowledge regarding social and communication skills, and the influence of the social context on 
occupational performance, they might be able to assist with addressing these concerns. The qualitative analysis indicated that communication was mentioned approximately 31 out of 170 instances (see Table 3). Specifically, social skills, group interaction, social connections with friends and family, journaling and communication logs were mentioned as potential countermeasures.

Performance patterns such as routines, roles, rituals, and habits appeared as a major theme in our qualitative analysis being mentioned 30 out of 170 instances. Specifically, sleep and rest routines were mentioned frequently. Because sleep and rest are a major area of research for space exploration, OT practitioners might provide significant insight into helping astronauts maintain regular sleep and rest routines to optimize cognition and task performance.

The qualitative analysis indicated that therapists believe adaptation to be extremely important for astronauts undergoing space travel. Adapting to a new environment focused on adapting to a difference sensory environment. This includes adapting to different roles or activities or preferred activities for participants in artificial environment as well as changes in gravity to occupations and tools. Being experts in adaptation and environmental modification, OT practitioners might be able to provide significant insight into helping astronauts adapt to the extreme environment experienced during space travel.

Interestingly, when asked what thoughts participants had about the role of OS in deep space exploration, a very small pool of participants included the term "occupational science" in their responses. These results indicate that the term or concept of OS does not easily come to mind for this population. It is unclear if this is a lack of knowledge of or familiarity with the concepts of OS. However, participants responded to this question multiple times with the terms "occupational deprivation" and "meaning," two concepts closely associated with OS.

\subsection{Limitations}

It is important to note that the list of occupational therapy interventions suggested from the respondents is by no means exhaustive. The responses suggest a role for occupational therapy as a means to address the many health risks of long duration space missions. The sample interventions collected by this survey are, at the very least, a foothold to occupational therapy's claim as an integral part of maintaining the health of people who participate in space missions.
Another limitation of our study was the relatively small sample size of occupational therapists who took our survey. Additionally, the majority of the participants were from the west coast of the United States making it difficult to generalize to occupational therapy OT practitioners across the United States, much less across the globe. Many of the participants also currently work in academia which might have also somewhat skewed results. Furthermore, it is unknown what prior knowledge the individuals who participated in the survey have regarding space exploration, which may also compromise the validity of results. Despite these limitations, this study provides an excellent foundation for future studies. Based on these findings, relevant frames of reference, models of practice and interventions have been identified that can assist future researchers in helping astronauts optimize health and well-being, resulting in enhanced task performance and mission success.

\subsection{Conclusion}

The OT profession firmly believes OS can inform the long duration space mission. Moreover, the OT profession believes OT interventions can be used by space travelers on long duration space flights. Occupations can either facilitate or limit the capacity of an individual to succeed in adapting to environmental demands. Deep space exploration presents environmental demands not yet experienced by humankind. By exploring the many dimensions and inherent meaning of occupations, insight into meaningful occupational therapy interventions in space can be illuminated. These interventions can address the deficits associated with long duration space exploration.

\section{Acknowledgments}

The researchers would like to thank Dr. Yvonne Cagle and Jan Kingaard, and all those involved with the Long Duration Space Exploration Behavioral Readiness Consortium 2030 for their guidance in the creation of this article. Each of the authors would like to thank their families and friends who have supported us throughout this process. We sincerely hope this paper will contribute to the knowledge bank for the Long Duration Space Exploration Behavioral Readiness Consortium 2030 and that elements of this paper will be recognized globally in the future. 


\section{Conflict of interest}

The authors have no conflict of interest to report.

\section{References}

[1] Bachmand K, Otto C, Leveton L. Countermeasures to mitigate the negative impact of sensory deprivation and social isolation in long-duration space flight. 2012. NASA Johnson Space Center, Houston, TX: National Science Biomedical Research Institute and USRA Division of Space Life Sciences.

[2] Canan JW. Health effects of human spaceflight. Aerosp Am 2013;24-30

[3] De la Torre G. Cognitive Neuroscience in Space. Life 2014;4(3):281-94

[4] Koppelmans V, Erdeniz B, De Dios Y, Wood S, ReuterLorenz P, Kofman I, et al. Study protocol to examine the effects of spaceflight and a spaceflight analog on neurocognitive performance: Extent, longevity, and neural bases. BMC Neurol 2013;13(205):1471-2377.

[5] Fulford MH. To infinity and beyond! Human spaceflight and life science. The FASEB J 2011;25(9):2858-64.

[6] Palinkas L, Keeton K, Shea C, Leveton L. Psychosocial characteristics of optimum performance in isolated and confined environments. Hanover, MD: NASA Center for AeroSpace Information; 2011.

[7] Ruff E. Psychological effects of space-flight. Aerosp Med Hum Perform 1961;32:639-42.

[8] Souvestre P, Landrock CK, Blaber AP. Reducing incapacitating symptoms during space flight: Is postural deficiency syndrome an applicable model? Hippokratia 2008;12(1): 41-8.

[9] Henderson A, Cermak, S, Coster, W, Murray, E, Trombly, C, Tickle-Degnen L. The issue is: Occupational science is multidimensional. American Journal of Occupational Therapy 1991;45:370-2.

[10] The American Occupational Therapy Association. The American Journal of Occupational Therapy 2014:68(1): $1-52$.

[11] Shaw L, Laliberte, D. Using occupational science to study occupational transitions in the realm of work. Work 2009;32:361-4

[12] Polanyi M, Tompa E. Rethinking work-health models for the new global economy: A qualitative analysis of emerging a emerging dimension of work. Work 2004;23(1):3-18.

[13] Schreuera N, Myhilla W, Aratan-Bergmana T, Samanta D, Blancka P. Occupational therapists as mediators in the interactive process. Work 2009;33:1-12

[14] Trudela L, Vonarx N, Simarda C, Freemana A, Vezina M, Brisson C, Alain V, Bourbonnais R, Dugas N. The adverse effects of psychological constraints at work. Work 2009;34:345-57

[15] Maxwell J. Qualitative research design: An interactive approach. Thousand Oaks, Calif,: Sage Publications; 1996.

[16] Patton M. Qualitative research and evaluation methods. Thousand Oaks, Calif.: Sage Publications; 2002.

[17] Seidman IE. Interviewing as qualitative research: A guide for researchers in education and the social sciences. New York, NY: Teachers College, 1991.

[18] Cowing K. Researching Changes To Astronaut Vision In Space-SpaceRef [Internet]. Spaceref.com.
2013 [cited 15 February 2015]. Available from: http://spaceref.com/space-medicine/researching-changesto-astronaut-vision-in-space.html

[19] Rasmussen J. (Ed.). Man in isolation and confinement Chicago, IL: Aldine Pub, 1997.

[20] Dawson D, Reid K. Fatigue, alcohol and performance impairment. Nature 1997;388(6639):235.

[21] Ferguson S, Lamond N, Kandelaars K, Jay S, Dawson D. The Impact of Short, Irregular Sleep Opportunities at Sea on the Alertness of Marine Pilots Working Extended Hours. Chronobiologia 2008;25(2-3):399-411.

[22] Leszczyńska I, Jeżewska M, Grubman-Nowak M. Dynamics of stress as a predictor of health consequences in Polish drilling platform workers. Longitudinal study: Part I. Int Marit Health 2014;65(1):33-40.

[23] Hall J, Cruser D, Podawiltz A, Mummert D, Jones H, Mummert M. Psychological stress and the cutaneous immune response: Roles of the HPA axis and the sympathetic nervous system in atopic dermatitis and psoriasis. Dermatol Res Pract 2012;2012:1-11.

[24] Ziegelstein RC. Acute emotional stress and cardiac arrhythmias. JAMA 2007;298(3):342-329.

[25] Ball R, Evans H. Safe Passage: Astronaut Care for Exploration Missions. Washington, D.C.: National Academy Press; 2001.

[26] Tooth L, McKenna K. Contemporary issues in cardiac rehabilitation: Implications for occupational therapists. $\mathrm{Br} \mathrm{J}$ Occup Ther 1996;59(3):133-40.

[27] Tomes H. Cardiac rehabilitation: An occupational therapist's perspective. Br J Occup Ther 1990;53(7):285-7.

[28] Bloomberg J, Peters B, Cohen H, Mulavara A. Enhancing astronaut performance using sensorimotor adaptability training. Front Syst Neurosci 2015;16(90):129

[29] Krueger WWO. Controlling motion sickness and spatial disorientation and enhancing vestibular rehabilitation with a user-worn see-through display. Laryngoscope 2010:121(2):17-35

[30] Trapp W, Landgrebe M, Hoesl K, Lautenbacher S, Gallhofer B, Gunther W, Hajak G. The effect of vestibular rehabilitation on adults with bilateral vestibular hypofunction: A systematic review. J Vestib Res 2012;22:5-6.

[31] Porciuncula F, Johnson CC, Glickman LB. The effect of vestibular rehabilitation on adults with bilateral vestibular hypofunction. J Vestib Res 2012;22(5-6):283-98.

[32] Cohen H, Kimball T. Changes in repetitive head movement task after vestibular rehabilitation. Clinical Rehabilitation. J Vestib Res 2004;(18):125-31.

[33] Balance Centers. OT Takes Leading Role in Vestibular Treatment. [Internet]. United States: Florida Balance Centers; 2015 Nov [Cited 2015 Nov 12]. Available from: http://www.balancecenters.com/?page_id=52

[34] Cohen S, Alford R. Use of the Vestibular Disorders Activities of Daily Living Scale to describe functional limitations in patients with vestibular disorders. J Vestib Res 2014;(24):33-8.

[35] Cole MB, Tufano R. Applied theories in occupational therapy: A practical approach. Thorofare, NJ: Slack, Inc. 2008.

[36] Cohen-Mansfield J, Werner P. Management of verbally disruptive behaviors in nursing home residents. J Gerontol A Biol Sci Med 1997;52(6):368-77.

[37] Nordvik E, Walle K, Nyberg K, Fjelle M, Walhovd B, Westlye T, Tornas S. Bridging the gap between clinical neuroscience and cognitive rehabilitation: The role of $\operatorname{cog}$ nitive training, models of neuroplasticity and advanced 
neuroimaging in future brain injury rehabilitation. Neurorehabilitation 2014;34(1):81-5.

[38] Cho M, Kim D, Chung J, Park J, You H, Yang Y. Effects of a cognitive-enhancement group training program on daily living activities, cognition, and depression in the demented elderly. J Phys Ther Sci 2015;27(3):681-4.

[39] Cochet A, Saoud M, Gabriele S, Borallier V, El Asmar C, Dalery J, D'Amato T. Impact of new cognitive remediation strategy on interpersonal problem solving skills and social autonomy in schizophrenia. Encephale 2005;32(2):189-95.

[40] Smith-Forbes ME, Najera C, Hawkins D. Combat operational stress control in Iraq and Afghanistan: Army occupational therapy. Mil Med 2014;179(3):279-84.

[41] Yazdi Z, Sadeghniiat-Haghighi K, Loukzadeh Z, Elmizadeh $\mathrm{K}$, Abbasi M. Prevalence of sleep disorders and their impacts on occupational performance: A comparison between shift workers and non-shift workers. Sleep Disord 2014:1-5.

[42] Leland NE, Marcione N, Schepens-Niemiec SL, Kelkar $\mathrm{K}$, Folgberg D. What is occupational therapy's role in addressing sleep problems among older adults? OTJR 2014;34(3):141-9.
[43] Lloyd C, Maas F. Occupational therapy group work in psychiatric settings. Br J Occup Ther 1997;60(5):226-30.

[44] Chen H, Tu H, Ho C. Understanding biophilia leisure as facilitating well-being and the environment: An examination of participants' attitudes toward horticultural activity. Leis Sci 2013;35(4):301-19.

[45] Kleiber D, Hutchinson S, Williams R. Leisure as a resource in transcending negative life events: Self-protection, self-restoration, and personal transformation. Leis Sci 2002;24(2):219-35

[46] Garcia M J, Castillo I, Queralt A. Leisure-time physical activity and psychological well-being in university students. Psychol Reports 2011;109(2):453-60

[47] Abraham A, Sommerhalde K, Abel T. Landscape and well-being: A scoping study on the impact of outdoor environments. Int J Public Health 2004;55(1):59-69.

[48] Foster J, Patel S. Human Research Roadmap [Internet]. Nasa.gov. 2015. Available from: http://humanresearch roadmap.nasa.gov/ 